\title{
Dark Equations and Their Light Integrability
}

Denis Blackmore, Anatolij K. Prykarpatski

To cite this article: Denis Blackmore, Anatolij K. Prykarpatski (2014) Dark Equations and Their Light Integrability, Journal of Nonlinear Mathematical Physics 21:3, 407-428, DOI: https://doi.org/10.1080/14029251.2014.936760

To link to this article: https://doi.org/10.1080/14029251.2014.936760

Published online: 04 January 2021 


\title{
Dark Equations and Their Light Integrability
}

\author{
Denis Blackmore \\ Department of Mathematical Sciences, New Jersey Institute of Technology, \\ Newark, New Jersey 07102-1982, United States of America \\ deblac@m.njit.edu \\ Anatolij K. Prykarpatski \\ Department of Applied Mathematics, AGH University of Science and Technology, \\ Krakow, Poland \\ pryk.anat@ua.fm,prykanat@cybergal.com
}

Received 27 January 2014

Accepted 8 May 2014

In memory of Boris Kupershmidt $(\dagger 2010)$, a mathematical light in the mysterious world of "dark" equations

\begin{abstract}
A relatively new approach to analyzing integrability, based upon differential-algebraic and symplectic techniques, is applied to some "dark equations "of the type introduced by Boris Kupershmidt. These dark equations have unusual properties and are not particularly well-understood. In particular, dark three-component polynomial Burgers type systems are studied in detail. Their matrix Lax representations are constructed, and the related symmetry recursion operators and infinite hierarchies of integrable nonlinear dynamical systems along with their Lax representations are derived. New linear Lax spectral problems for dark integrable countable hierarchies of dynamical systems are proposed and some special cases are considered as a means of indicating that the approach presented is applicable to a far wider class of dark equations than analyzed here.
\end{abstract}

Keywords: Burgers type system; differential-algebraic approach; asymptotic analysis; conserved quantities; Lax integrability, symmetry recursion operator; commuting infinite hierarchies of dynamical systems

2000 Mathematics Subject Classification: 35A30, 35G25, 35N10, 37K35, 58J70,58J72

\section{Introduction}

Boris Kupershmidt wrote in [22] , “... observing the Universe, astronomers have concluded that the motion of the stars can not be accounted for unless one assumes that most of the mass in the Universe is carried on by a 'dark matter' so far impervious to all attempts at being detected. There is now a similar concept of 'dark energy'. I shall discuss a different subject, 'dark equations' . These have never indicated that they influence anything or even exist, but if one supposes that they do exist, one can systematically discover them and study their properties, some of which turn out to be strange and mysterious...."

Amongst such dark equations, B. Kupershmidt singled out the dispersive equations $[43,46]$ of hydrodynamic type, discovered by B. Riemann in 1860 [40], whose generalized three-component 
representatives are

$$
\begin{aligned}
& u_{t}=-2 u u_{x}+h_{x}, \\
& h_{t}=-(u h)_{x}+u_{x x}+v_{x}, \\
& v_{t}=-(u v)_{x},
\end{aligned}
$$

and

$$
\begin{aligned}
& u_{t}=-2 u u_{x}+u_{x x}+h_{x}, \\
& h_{t}=-(u h)_{x}+v_{x}, \\
& v_{t}=-(u v)_{x},
\end{aligned}
$$

defined on a smooth functional manifold $M \subset C^{\infty}\left(\mathbb{R} ; \mathbb{R}^{3}\right)$, where $(u, h, v)^{\top} \in M$. We note that (1.1) is a natural extension of a system analyzed by Kupershmidt [22] and (1.2) appeared in [26] as a special case of a countable hierarchy of Burgers type multi-component systems possessing a special two-dimensional matrix Lax representation, whose structure in the 2-component case was recently explained in [3]. With $v=0$, the flow (1.2) was also studied in [11], where the corresponding symmetry recursion operator was constructed. In particular, in $[11,22,26]$ the dark integrability of such dispersive equations of hydrodynamic type was demonstrated, owing in part to the fact that the corresponding recursion operators are not always factorized by Poisson structures. The same property was also recently analyzed in $[3,37]$, where it was shown using differential-algebraic tools that for $v=0$ the flow (1.2)

$$
\begin{aligned}
& u_{t}=-2 u u_{x}+u_{x x}+h_{x}, \\
& h_{t}=-(u h)_{x},
\end{aligned}
$$

has only two local conserved quantities and, when $h=0$, this yields the Burgers hydrodynamic system

$$
u_{t}=-2 u u_{x}+u_{x x}
$$

which has just one local conserved quantity. Yet it has a nontrivial countable hierarchy of nonlocal conserved quantities. Moreover, the corresponding squared symmetry recursion operator has a nonstandard Poisson structure factorization, making it possible to generate $[1,4,36,39]$ a countable hierarchy of mutually commuting Hamiltonian systems. Other examples of dark equations are the Broer-Kaup-Kupershmidt

$$
\begin{aligned}
& u_{t}=-u u_{x}+u_{x x}-h_{x}, \\
& h_{t}=-(u h)_{x}-h_{x x}
\end{aligned}
$$

and the Kaup-Boussinesq

$$
\begin{aligned}
& u_{t}=-2 u u_{x}+h_{x} / 2, \\
& h_{t}=-2(u h)_{x}+u_{x x x} / 2,
\end{aligned}
$$

dispersive hydrodynamic flows, whose integrability and soliton-like solutions were investigated in $[5,22,23,30,36]$. Related multi-component dark-type extensions of (1.5) and (1.6) were recently constructed in [8]. 
We have also obtained Lax representations for some new dark integrable Kaup-Broer type

$$
\begin{aligned}
& u_{t}=-2 u_{x x}+8 u u_{x}+8(h u)_{x}+4 h h_{x}-4 h_{x x}, \\
& h_{t}=2 h_{x x}+4 u h_{x}+4 h h_{x},
\end{aligned}
$$

and

$$
\begin{gathered}
u_{t}=-u u_{x}+h_{x x x}, \quad u_{t}=-u u_{x}-2 h^{-1} u_{x x}, \\
h_{t}=-u_{x} h-u h_{x}, \quad h_{t}=-u_{x} h-u h_{x},
\end{gathered}
$$

Burgers type dynamical systems, both of which have only finitely many constants of motion, yet possess many-component bi-Hamiltonian completely integrable generalizations.

In the sequel, we shall understand "dark equations" as nonlinear Lax integrable dynamical systems that have hidden symmetries and related properties such as only finitely many local conservation laws, mixed dissipative and strongly dispersive properties, and unfactored symmetry recursion operators. Another possible characterization consistent with B. Kupershmidt's work is that an equation is "dark" if it is linearizable, has a finite number of local conservation laws and admits a Lax pair. In the interest of understanding these systems, we examined the flows (1.1) and (1.2) using a novel combination of the gradient-holonomic $[1,5,36]$ and differential-algebraic tools $[2,3,13-16,37]$ and proved they possess only a finitely many local conserved quantities. The standard corresponding symmetry recursion operators do not allow Poisson factorization, yet they generate countable hierarchies of mutually commuting nonlinear dynamical systems on $M$.

A key element in our analysis is determining the Nöther-Lax equation

$$
d \varphi / d t+K^{\prime, *} \varphi=0
$$

for a vector $\varphi \in T^{*}(M) \otimes \mathbb{C}$ subject to a given nonlinear dynamical system

$$
\frac{d}{d t}(u, h, v)^{\top}=K[u, h, v]
$$

where $K: M \rightarrow T(M)$ is the corresponding smooth vector field on $M$ and $K^{\prime, *}: T^{*}(M) \otimes \mathbb{C} \rightarrow$ $T^{*}(M) \otimes \mathbb{C}$ is the adjoint linear operator on the complexified cotangent space $T^{*}(M) \otimes \mathbb{C}$ to the Fréchet derivative $K^{\prime}: T(M) \otimes \mathbb{C} \rightarrow T(M) \otimes \mathbb{C}$ with respect to the standard bilinear conjugation form $(\cdot, \cdot)$ on $T^{*}(M) \otimes \mathbb{C} \times T(M) \otimes \mathbb{C}$.

We shall analyze the dark integrability properties of the Burgers systems (1.1) and (1.2) in detail to examine their Lie-algebraic and functional-analytic structures, but this is not the most important aspect of our work here. It is, in fact, to demonstrate how our approach can provide an effective and efficient means of analyzing a much wider class of dark equations.

\section{Differential-algebraic preliminaries}

For completeness we include some differential-algebraic preliminaries [1,14-18]. We start with the ring $\mathscr{K}:=\mathbb{R}\{\{x, t\}\},(x, t) \in \mathbb{R} \times(0, T)$, of convergent germs of real-valued smooth functions from $C^{\infty}\left(\mathbb{R}^{2} ; \mathbb{R}\right)$ and construct the associated differential quotient ring $\mathscr{K}\{u, h, v\}:=\operatorname{Quot}(\mathscr{K}[\Theta u, \Theta v])$ with respect to the functional variables $u, h, v \in \mathscr{K}$, where $\Theta$ denotes $[14,17,21,41,41]$ the standard monoid of all commuting differentiations $D_{x}$ and $D_{t}$, satisfying the usual Leibniz condition, and 
defined by

$$
D_{x}(x)=1=D_{t}(t), \quad D_{t}(x)=0=D_{x}(t),
$$

The ideal $I\{u, h, v\} \subset \mathscr{K}\{u, h, v\}$ is called differential if $I\{u, h, v\}=\Theta I\{u, h, v\}$. In the differential ring $\mathscr{K}\{u, h, v\}$, interpreted as an invariant differential ideal in $\mathscr{K}$, there are two naturally defined differentiations

$$
D_{t}, D_{x}: \mathscr{K}\{u, h, v\} \rightarrow \mathscr{K}\{u, h, v\},
$$

satisfying the commutation relationship

$$
\left[D_{t}, D_{x}\right]=0 .
$$

Consider the ring $\mathscr{K}\{u, h, v\}, u, h, v \in \mathscr{K}$, and the exterior differentiation $d: \mathscr{K}\{u, h, v\} \rightarrow$ $\Lambda^{1}(\mathscr{K}\{u, h, v\}),: d: \Lambda^{p}(\mathscr{K}\{u, h, v\}) \rightarrow \Lambda^{p+1}(\mathscr{K}\{u, h, v\})$ for $p \in \mathbb{Z}_{+}$, acting in the freely generated Grassmann algebras $\Lambda(\mathscr{K}\{u, h, v\})=\oplus_{p \in \mathbb{Z}_{+}} \Lambda^{p}(\mathscr{K}\{u, h, v\})$ over the field $\mathbb{C}$, where

$$
\begin{aligned}
\Lambda^{1}(\mathscr{K}\{u, h, v\}):= & \mathscr{K}\{u, h, v\} d x+\mathscr{K}\{u, h, v\} d t+\sum_{j, k \in \mathbb{Z}_{+}} \mathscr{K}\{u, h, v\} d u^{(j, k)}+ \\
+ & \sum_{j, k \in \mathbb{Z}_{+}} \mathscr{K}\{u, h, v\} d h^{(j, k)}+\sum_{j, k \in \mathbb{Z}_{+}} \mathscr{K}\{u, h, v\} d v^{(j, k)} \\
& u^{(j, k)}:=D_{t}^{j} D_{x}^{k} u, \quad h^{(j, k)}:=D_{t}^{j} D_{x}^{k} h, v^{(j, k)}:=D_{t}^{j} D_{x}^{k} v, \\
& \Lambda^{2}(\mathscr{K}\{u, h, v\}):=\mathscr{K}\{u, h, v\} d \Lambda^{1}(\mathscr{K}\{u, h, v\}), \ldots, \\
& \Lambda^{p+1}(\mathscr{K}\{u, h, v\}):=\mathscr{K}\{u, h, v\} d \Lambda^{p}(\mathscr{K}\{u, h, v\}),
\end{aligned}
$$

The triple $\mathscr{A}:=(\mathscr{K}\{u, h, v\}, \Lambda(\mathscr{K}\{u, h, v\}) ; d)$ will be called the Grassmann differential algebra with generators $u, h, v \in \mathscr{K}$. In the algebra $\mathscr{A}$, generated by $u, h, v \in \mathscr{K}$, one naturally defines the action of differentiations $D_{t}, D_{x}$ and $\partial / \partial u^{(j, k)}, \partial / \partial h^{(j, k)}, \partial / \partial v^{(j, k)}: \mathscr{A} \rightarrow \mathscr{A}, j, k \in \mathbb{Z}_{+}$, as follows:

$$
\begin{aligned}
& D_{t} u^{(j, k)}=u^{(j+1, k)}, D_{x} u^{(j, k)}=u^{(j, k+1)}, \\
& D_{t} h^{(j, k)}=h^{(j+1, k)}, D_{x} h^{(j, k)}=h^{(j, k+1)}, \\
& D_{t} v^{(j, k)}=v^{(j+1, k)}, D_{x} v^{(j, k)}=v^{(j, k+1)} \text {, } \\
& D_{t} d u^{(j, k)}=d u^{(j+1, k)}, D_{x} d u^{(j, k)}=d u^{(j, k+1)}, \\
& D_{t} d h^{(j, k)}=d h^{(j+1, k)}, D_{x} d h^{(j, k)}=d h^{(j, k+1)}, \\
& D_{t} d v^{(j, k)}=d v^{(j+1, k)}, D_{x} d v^{(j, k)}=d v^{(j, k+1)}, \\
& d P[u, h, v, h]=\sum_{j, k \in \mathbb{Z}_{+}}( \pm) \partial P[u, h, v, h] / \partial u^{(j, k)} \wedge d u^{(j, k)}+ \\
& +\sum_{j, k \in \mathbb{Z}_{+}}( \pm) \partial P[u, h, v, h] / \partial h^{(j, k)} \wedge d h^{(j, k)}+ \\
& +\sum_{j, k \in \mathbb{Z}_{+}}( \pm) \partial P[u, h, v, h] / \partial v^{(j, k)} \wedge d v^{(j, k)}:=<P^{\prime}[u, h, v], \wedge(d u, d h, d v)^{\top}>_{\mathbb{C}^{2}},
\end{aligned}
$$

where $\wedge$ denotes the standard [18] exterior multiplication in $\Lambda(\mathscr{K}\{u, h, v\})$, and for any $P[u, h, v] \in$ $\Lambda(\mathscr{K}\{u, h, v\})$ the mapping

$$
P^{\prime}[u, h, v] \wedge: \Lambda^{0}(\mathscr{K}\{u, h, v\})^{3} \rightarrow \Lambda(\mathscr{K}\{u, h, v\}),
$$

is linear. Moreover, the commutation relationships

$$
D_{x} d=d D_{x}, \quad D_{t} d=d D_{t}
$$

hold in the Grassmann differential algebra $\mathscr{A}$. The following remark [17] is also important.

Remark 2.1. Any Lie derivative $L_{V}: \mathscr{K}\{u, h, v\} \rightarrow \mathscr{K}\{u, h, v\}$ with $L_{V}: \mathscr{K} \subset \mathscr{K}$, can be uniquely extended to the differentiation $L_{V}: \mathscr{A} \rightarrow \mathscr{A}$, satisfying $L_{V} d=d L_{V}$. 
The variational derivative, or the functional gradient $\nabla P[u, h, v] \in \Lambda(\mathscr{K}\{u, h, v\})^{3}$ with respect to $u, h, v \in \mathscr{K}$, is defined for any $P[u, h, v] \in \Lambda(\mathscr{K}\{u, h, v\})$ by

$$
\operatorname{grad} P[u, h, v]=P^{\prime, *}[u, h, v](1),
$$

where a mapping $P^{\prime, *}[u, h, v]: \Lambda^{0}(\mathscr{K}\{u, h, v\}) \rightarrow \Lambda^{0}(\mathscr{K}\{u, h, v\})^{3}$ is the formal adjoint for that of (2.5). This is based on the following result for a special case in [13-17,31].

Lemma 2.1. Let the differentiations $D_{x}$ and $D_{t}: \Lambda(\mathscr{K}\{u, h, v\}) \rightarrow \Lambda(\mathscr{K}\{u, h, v\})$ satisfy (2.5). Then the mapping

$\operatorname{Kergrad} /(\operatorname{Im} d \oplus \mathbb{C}) \simeq H^{1}(\mathscr{A}):=\operatorname{Ker}\left\{d: \Lambda^{1}(\mathscr{K}\{u, h, v\}) \rightarrow \Lambda^{2}(\mathscr{K}\{u, h, v\})\right\} / d \Lambda^{0}(\mathscr{K}\{u, h, v\})$

is a canonical isomorphism, where $H^{1}(\mathscr{A})$ is the cohomology class of the Grassmann complex $\Lambda(\mathscr{K}\{u, h, v\})$.

It is well known [41] that for $\mathscr{K}\{u, h, v\}$ not all of the cohomology classes $H^{j}(\mathscr{A}), j \in \mathbb{Z}_{+}$, are trivial. However, one can impose additional restrictions on $u, h, v \in \mathscr{K}$, which give rise to the condition $H^{1}(\mathscr{A})=0$, or equivalently, to the relationship $\operatorname{Ker} \nabla=\operatorname{Im} D_{x} \oplus \operatorname{Im} D_{t} \oplus \mathbb{C}$. In addition, the following simple relationship holds:

$$
\operatorname{grad}\left(\operatorname{Im} D_{x} \oplus \operatorname{Im} D_{t}\right)=0 .
$$

Using Lemma 2.1, we define the equivalence class $\widetilde{\mathscr{A}}:=\mathscr{A} /\left\{\operatorname{Im} D_{x} \oplus \operatorname{Im} D_{t} \oplus \mathbb{R}\right\}$ : $=\mathscr{D}(\mathscr{A} ; d x d t)$ of functionals; that is, any element $\gamma \in \mathscr{D}(\mathscr{A} ; d x d t)$ can be represented as an integral $\gamma:=\iint d x d t \gamma[u, h, v] \in \mathscr{D}(\mathscr{A} ; d x d t)$ for some $\gamma[u, h, v] \in \Lambda(\mathscr{K}\{u, h, v\})$ with respect to the Lebesgue measure $d x d t$ on $\mathbb{R}^{2}$.

Consider now our two-component dynamical system (1.1) as the polynomial differential constraint

$$
D_{t}(u, h, v)^{\top}=K[u, h, v]
$$

imposed on the ring $\mathscr{K}\{u, h, v\}$. The following definitions will be useful for our further analysis.

Definition 2.1. Let the reduced ring $\overline{\mathscr{K}}\{u, h, v\}:=\left.\mathscr{K}\{u, h, v\}\right|_{D_{t}(u, h, v)^{\top}=K[u, h, v]}$. Then the triple $\overline{\mathscr{A}}:=(\overline{\mathscr{K}}\{u, h, v\}, \Lambda(\overline{\mathscr{K}}\{u, h, v\}), d)$ will be called a reduced Grassmann differential algebra over the reduced ring $\overline{\mathscr{K}}\{u, h, v\}$.

Definition 2.2. Any pair of elements $(\gamma[u, h, v], \rho[u, h, v])^{\top} \in \Lambda^{0}(\overline{\mathscr{K}}\{u, h, v\})^{2}$, satisfying the relationship

$$
D_{t} \gamma[u, h, v]+D_{x} \rho[u, h, v]=0,
$$

is called a scalar conserved quantity with respect to $D_{x}$ and $D_{t}$.

In this setting, one can define the spaces of functionals $\mathscr{D}(\overline{\mathscr{A}} ; d x):=\overline{\mathscr{A}} /\left\{D_{x} \overline{\mathscr{A}}\right\}$ and $\mathscr{D}(\overline{\mathscr{A}} ; d t)=\overline{\mathscr{A}} /\left\{D_{t} \overline{\mathscr{A}}\right\}$ on the the reduced Grassmann differential algebra $\overline{\mathscr{A}}$. From the functional point of view, these factor spaces $\mathscr{D}(\overline{\mathscr{A}} ; d x)$ and $\mathscr{D}(\overline{\mathscr{A}} ; d t)$ can be understood more classically as the corresponding spaces of suitably defined integral expressions subject to the measures $d x$ and $d t$, respectively. Then (2.12) means that $\gamma:=\int d x \gamma[u, h, v] \in \mathscr{D}(\overline{\mathscr{A}} ; d x)$ is a conserved quantity for $D_{t}$, and $\rho:=\int d t \rho[u, h, v] \in \mathscr{D}(\overline{\mathscr{A}} ; d t)$ is a conserved quantity for $D_{x}$. 
Since (2.11) defines $[17,18]$ on the reduced ring $\overline{\mathscr{K}}\{u, h, v\}$ a smooth vector field $K$ : $\overline{\mathscr{K}}\{u, h, v\} \rightarrow T(\overline{\mathscr{K}}\{u, h, v\})$, one can construct the corresponding Lie derivative $L_{K}: \overline{\mathscr{A}} \rightarrow \overline{\mathscr{A}}$ along this vector field and calculate the differential expression

$$
\partial \varphi[u, h, v] / \partial t+L_{K} \varphi[u, h, v]=0
$$

for the element $\varphi[u, h, v]:=\operatorname{grad} \gamma[u, h, v] \in \Lambda^{0}(\overline{\mathscr{K}}\{u, h, v\})^{3}$, where $\gamma \in \mathscr{D}(\overline{\mathscr{A}} ; d x)$ is an arbitrary scalar conserved quantity with respect to $D_{t}$. One easily calculates that [36] in $\overline{\mathscr{K}}\{u, h, v\}$ the following Lax relationship is identically satisfied [24], and this leads to the classical Nöther lemma $[1,31,36]$.

Lemma 2.2. (Nöther-Lax) Let a quantity $\varphi[u, h, v] \in \Lambda^{0}(\overline{\mathscr{K}}\{u, h, v\})^{3}$ be such that

$$
D_{t} \varphi[u, h, v]+K^{\prime, *}[u, h, v] \varphi[u, h, v]=0,
$$

which is equivalent to (2.13), holds in $\overline{\mathscr{K}}\{u, h, v\}$ satisfying the differential constraint (2.11). Then, if the Volterra condition $\varphi^{\prime, *}[u, h, v]=\varphi^{\prime}[u, h, v]$ is satisfied in the $\overline{\mathscr{K}}\{u, h, v\}$, the homology functional

$$
\gamma:=\int_{0}^{1} d \lambda \int d x<\varphi[\lambda u, \lambda h, \lambda v],(u, h, v)^{\top}>_{\mathbb{C}^{3}} \in \mathscr{D}(\overline{\mathscr{A}} ; d x)
$$

is a scalar conserved quantity with respect to $D_{t}$.

Assume now that the system (2.11) possesses a nontrivial differential Lax representation in the matrix form

$$
D_{x} f(x, t ; \lambda)=l[u, h, v ; \lambda] f(x, t ; \lambda),
$$

compatible with the adjoint matrix evolution equation

$$
D_{t} f(x, t ; \lambda)=p(l) f(x, t ; \lambda)
$$

for some matrices $l:=l[u, h, v ; \lambda]$ and $p(l) \in E n d \Lambda^{0}\left(\overline{\mathscr{K}}\left\{u, h, v ; D_{x}^{-1} \sigma \mid N\right\}\right)^{n}$, acting in a vector space of functions of fixed dimension $n \in \mathbb{N}, f(x, t ; \lambda) \in \Lambda^{0}\left(\overline{\mathscr{K}}\left\{u, h, v ; D_{x}^{-1} \sigma \mid N\right\}\right)^{n}$, analytically depending on the parameter $\lambda \in \mathbb{C}$, where $\overline{\mathscr{K}}\left\{u, h, v ; D_{x}^{-1} \sigma \mid N\right\}, N \in \mathbb{Z}_{+}$, is a nonlocal finitely extended differential ring $\overline{\mathscr{K}}\{u, h, v\}$. Then the following result (based on the gradient-holonomic approach $[1,5])$ holds.

Proposition 2.1. The Lax integrable dynamical system (2.11) possesses a countable hierarchy (either finite or infinite) of naturally ordered functionally independent scalar conserved differential quantities

$$
D_{t} \sigma_{j}[u, h, v]+D_{x} \rho_{j}[u, h, v]=0,
$$

where the pairs $\left(\sigma_{j}[u, h, v], \rho_{j}[u, h, v]\right)^{\top} \in \Lambda^{0}(\overline{\mathscr{K}}\{u, h, v\})^{2}, j \in \mathbb{Z}_{+}$.

Proof. Assume that the Lax integrable dynamical system (2.11) possesses a countable set of naturally ordered functionally independent scalar conserved quantities (2.18). Let $\overline{\mathscr{K}}\left\{u, h, v ; D_{x}^{-1} \sigma \mid N\right\}$ 
denote the finitely extended differential ring $\mathscr{K}\left\{u, h, v ;\left\{D_{x}^{-1} \sigma_{j}[u, h, v]: 0 \leq j \leq N\right\}\right\}$ for an arbitrary $N \in \mathbb{Z}_{+}$under the constraints (2.11). Then the Lax equation (2.14) considered on the invariant functional submanifold

$$
\begin{aligned}
& M_{N}:=\left\{(u, h, v)^{\top} \in M: \quad \operatorname{grad}<c^{(N)}, \int d x \sigma^{(N)}>\mathbb{C}^{N+1}=0,\right. \\
& \left.c^{(N)} \in \mathbb{C}^{N+1} \backslash\{0\}, \sigma^{(N)}:=\left(\sigma_{0}, \sigma_{1}, \ldots, \sigma_{N}\right)^{\top} \in \Lambda^{0}(\overline{\mathscr{K}}\{u, h, v\})^{N+1}\right\},
\end{aligned}
$$

allows [1,36] a special solution $\varphi(x ; \lambda) \in \Lambda^{0}\left(\overline{\mathscr{K}}\left\{u, h, v ; D_{x}^{-1} \sigma \mid N\right\}\right)^{3}$ in the form

$$
\varphi(x ; \lambda) \sim \psi(x, t ; \lambda) \exp \left\{\omega(t ; \lambda)+D_{x}^{-1} \sigma(x, t ; \lambda)\right\}
$$

with a scalar analytical "dispersion" function $\omega(t ; \cdot): \mathbb{C} \rightarrow \mathbb{C}$, determined for all $t \in[0, T)$, where a vector $\psi \in \Lambda^{0}(\overline{\mathscr{K}}\{u, h, v\})^{3}$ and element $\sigma(x, t ; \lambda) \in \Lambda^{0}(\overline{\mathscr{K}}\{u, h, v\})$ possess the following asymptotic expansions

$$
\sigma(x, t ; \lambda) \sim \sum_{j \in \mathbb{Z}_{+}} \sigma_{j}[u, h, v] \lambda^{-j+|\sigma|}, \psi(x, t ; \lambda) \sim \sum_{j \in \mathbb{Z}_{+}} \psi_{j}[u, h, v] \lambda^{-j+|\psi|}
$$

as $|\lambda| \rightarrow \infty$ for some fixed $|\sigma|,|\psi| \in \mathbb{Z}_{+}$. Moreover, owing to (2.14), all of the scalar functionals

$$
\gamma_{j}:=\int d x \sigma_{j}[u, h, v]
$$

for $j \in \mathbb{Z}_{+}$are conserved quantities with respect to $D_{t}$. Now, conversely, if (2.14) possesses an asymptotic (as $|\lambda| \rightarrow \infty)$ solution in the form (2.20) $\varphi[u, h, v ; \lambda] \in \Lambda^{0}\left(\overline{\mathscr{K}}\left\{u, h, v ; D_{x}^{-1} \sigma \mid N\right\}^{3}\right.$ with compatible expansions (2.21), then all of the scalar functionals (2.22) are, a priori, the conserved quantities with respect to the $D_{t}$. That is, there exist scalars $\rho_{j}[u, h, v] \in \Lambda^{0}(\overline{\mathscr{K}}\{u, h, v\}), j \in \mathbb{Z}_{+}$, satisfying (2.18). The analytical expressions for representation (2.20) and asymptotic expansions (2.21) for a Lax integrable dynamical system (2.11) readily follow both from the general theory of asymptotic solutions $[9,42]$, applied to the linear matrix differential system $(2.16)$, and from the fact $[1,10,29,36]$ that the trace functional $\Delta[u, h, v ; \lambda]:=\operatorname{tr} S(x ; \lambda) \in \mathscr{D}(\overline{\mathscr{A}} ; d x) \cap \mathscr{D}(\overline{\mathscr{A}} ; d t)$ is for almost all $\lambda \in \mathbb{C}$ a conserved quantity with respect to both $D_{t}$ and $D_{x}$. Here the expression

$$
S(x ; \lambda):=F(x, t ; \lambda) C(\lambda) \tilde{F}^{\top}(x, t ; \lambda) \in \Lambda^{0}\left(\overline{\mathscr{K}}\left\{u, h, v ; D_{x}^{-1} \sigma \mid N\right\}\right)^{n}
$$

satisfies the important characteristic matrix differential relationship

$$
D_{x} S(x ; \lambda)=[l[u, h, v ; \lambda], S(x ; \lambda)],
$$

and is defined by means of some constant matrix $C(\lambda) \in$ End $\mathbb{C}^{n}$ and of the fundamental solutions $F(x, t ; \lambda)$ and $\tilde{F}(x, t ; \lambda) \in E n d \Lambda^{0}\left(\overline{\mathscr{K}}\left\{u, h, v ; D_{x}^{-1} \sigma \mid N\right\}\right)^{n},(x, t) \in \mathbb{R} \times[0, T)$, to

$$
D_{x} f(x, t ; \lambda)=l[u, h, v ; \lambda] f(x, t ; \lambda)
$$

and, respectively, its adjoint

$$
D_{x} \tilde{f}(x, t ; \lambda)=-l[u, h, v ; \lambda]^{\top} \tilde{f}(x, t ; \lambda),
$$

where $f(x, t ; \lambda), \tilde{f}(x, t ; \lambda) \in \Lambda^{0}\left(\overline{\mathscr{K}}\left\{u, h, v ; D_{x}^{-1} \sigma \mid N\right\}\right)^{n}$. Consequently [1,36],

$$
\operatorname{grad} \Delta(\lambda)[u, h, v]:=\varphi[u, h, v ; \lambda]=\operatorname{tr}\left(l^{\prime, *} S\right)[u, h, v ; \lambda]
$$

owing to Lemma 2.2 a priori satisfies the Lax equation (2.14). Then desired result then follows from the asymptotic properties of linear equations (2.25) and (2.26). 
An important consequence of Proposition 2.1 is that (2.24) and (2.27) make it possible, via their analytical structure, to retrieve the a priori unknown linear Lax spectral problem (2.16) and next to apply it to finding exact solutions to (2.11) by means of the inverse spectral transform method [7, 10, 28,29] and the related algebro-geometric [29] tools and techniques.

\section{Three-component polynomial Burgers systems and their integrability}

\subsection{Integrability analysis of the dispersive hydrodynamic flow (1.1)}

We now analyze the Lax integrability of the dynamical system (1.1). To do this using the above approach, we must prove that the Lax equation (2.14) has an asymptotic solution of the form (2.20) in $\overline{\mathscr{K}}\left\{u, h, v ; D_{x}^{-1} \sigma \mid N\right\}^{3}$.

Proposition 3.1. The Lax equation (6) with the differential matrix operator

$$
K^{\prime, *}[u, h, v]=\left(\begin{array}{ccc}
2 u D_{x} & h D_{x}+D_{x}^{2} v D_{x} \\
-D_{x} & u D_{x} & 0 \\
0 & -D_{x} & u D_{x}
\end{array}\right),
$$

possesses the asymptotic solution

$$
\varphi(x ; \lambda)=(1 / \lambda, 1, \lambda)^{\top} g(x ; \lambda) \exp \left[-\lambda^{3} t-\lambda^{2} x-D_{x}^{-1}\left(u \lambda+h+\lambda^{-1} v\right)\right]
$$

as $|\lambda| \rightarrow \infty$, where the scalar invertible element

$$
g(x ; \lambda):=\exp \left(-u \lambda^{-1}+\left(u^{2} / 2-h\right) \lambda^{-2}+\sum_{j \in \mathbb{Z}_{+} \backslash\{0,1,2\}} D_{x}^{-1} \sigma_{j}[u, h, v] / \lambda^{j}\right) \in \Lambda^{0}(\overline{\mathscr{K}}\{u, h, v\}) .
$$

The solution (3.2) corresponds to the local conserved quantity $\Delta(\lambda):=\int d x\left(u \lambda+h+\lambda^{-1} v\right) \in$ $\mathscr{D}(\overline{\mathscr{A}} ; d x)$ in the finitely extended ring $\overline{\mathscr{K}}\left\{u, h, v ; D_{x}^{-1} \sigma \mid 3\right\}$ :

$$
\operatorname{grad} \Delta(\lambda)[u, h, v]=\varphi(x ; \lambda) \in \overline{\mathscr{K}}\left\{u, h, v ; D_{x}^{-1} \sigma \mid 3\right\}^{3} .
$$

Proof. Assume that the Lax equation (2.14) possesses the asymptotic solution (2.20) as $|\lambda| \rightarrow \infty$, where $\omega(x, t ; \lambda)=-\lambda^{3} t-\lambda^{2} t$,

$$
\varphi(x ; \lambda)=\psi(x, t ; \lambda) \exp \left\{-\lambda^{3} t-\lambda^{2} x+D_{x}^{-1} \sigma(x, t ; \lambda)\right\}
$$

and

$$
\psi(x, t ; \lambda)=(1, a(x, t ; \lambda), b(x, t ; \lambda))^{\top},
$$

which reduces to an equivalent system of the differential-functional relationships

$$
\begin{aligned}
a D_{x}^{-1} d \sigma / d t+d a / d t-\lambda^{3} a+2 u \sigma_{x}+2 u a \sigma-2 u \lambda^{2} a-h \lambda^{2} & \\
+h \sigma+\sigma_{x}+\lambda^{4}-2 \lambda^{2} \sigma+\sigma^{2}+v b_{x}+v b \sigma-\lambda^{2} v b & =0 ; \\
D_{x}^{-1} d \sigma / d t-\lambda^{3}-a_{x}-a \sigma-\lambda^{2}+u \sigma-\lambda^{2} u & =0 ; \\
d b / d t-\lambda^{3} b+b D_{x}^{-1} d \sigma / d t-\sigma+\lambda^{2}+u b_{x}+u b \sigma-\lambda^{2} u b & =0 .
\end{aligned}
$$

The coefficients of the corresponding asymptotic expansions

$$
a(x, t ; \lambda) \sim \sum_{j \geq-1} a_{j}[u, h, v] \lambda^{-j}, b(x, t ; \lambda) \sim \sum_{j \geq 0} b_{j}[u, h, v] \lambda^{-j}, \sigma(x, t ; \lambda) \sim \sum_{j \geq-1} \sigma_{j}[u, h, v] \lambda^{-j}
$$


as $|\lambda| \rightarrow \infty$ should satisfy three countable hierarchies of the recurrent relationships

$$
\begin{gathered}
\sum_{k \in \mathbb{Z}_{+}} a_{j-k} D_{x}^{-1} d \sigma_{k} / d t+d a_{j} / d t-a_{j+3}+2 u \sigma_{j, x}+2 u \sum_{k \in \mathbb{Z}_{+}} a_{j-k} \sigma_{k}- \\
-2 u^{2} a_{j+2}+h \sigma_{j}-h \delta_{j,-2}+\sigma_{j, x}+\delta_{j,-4}-2 \sigma_{j+2}+\sum_{k \in \mathbb{Z}_{+}} \sigma_{j-k} \sigma_{k}+ \\
+v b_{j, x}+v \sum_{k \in \mathbb{Z}_{+}} b_{j-k} \sigma_{k}-v b_{j+2}=0 ; \\
D_{x}^{-1} d \sigma_{j} / d t-\delta_{j,-3}-a_{j, x}-\sum_{k \in \mathbb{Z}_{+}} a_{j-k} \sigma_{k}+a_{j+2}+u a_{j}-u \delta_{j,-2}=0 ; \\
d b_{j} / d t-b_{j+3}+\sum_{k \in \mathbb{Z}_{+}} b_{j-k} D_{x}^{-1} \sigma_{k, t}-\sigma j+\delta_{j,-2}+ \\
+u b_{j, x}+u \sum_{k \in \mathbb{Z}_{+}} b_{j-k} \sigma_{k}-u b_{j+2}=0,
\end{gathered}
$$

compatible for all $j \in \mathbb{Z}_{+} \cup\{-4,-3,-2,-1\}$. It is easy to calculate from (3.9) the corresponding coefficients

$$
\begin{aligned}
\sigma_{-1} & =u, \sigma_{0}=-h, \sigma_{1}=-u_{x}-v, \\
\sigma_{2} & =-h_{x}+u u_{x}, \quad \sigma_{j+2}=D_{x} r_{j, x}, \ldots, \\
a_{-1} & =1, \quad a_{0}=0, \quad a_{1}=1, \quad a_{2}=0, \quad a_{3}=0, \ldots, a_{j}=0, \ldots ; \\
b_{1} & =1, \quad b_{2}=0, b_{3}=0, \ldots, b_{j}=0, \ldots
\end{aligned}
$$

for some local functionals $r_{j} \in \Lambda^{0}(\overline{\mathscr{K}}\{u, h, v\}), j \in \mathbb{Z}_{+}$, and conclude that only three functionals

$$
\begin{aligned}
\gamma_{-1} & :=\int d x \sigma_{-1}[u, h, v]=\int d x u, \gamma_{1}:=\int d x \sigma_{1}[u, h, v]=\int d x h, \\
\gamma_{2} & :=\int d x \sigma_{2}[u, h, v]=\int d x v
\end{aligned}
$$

are nontrivial conserved quantities with respect to $D_{t}$, since all other functionals for $j \in \mathbb{Z}_{+} \backslash\{0,1,2\}$

$$
\gamma_{j}:=\int d x \sigma_{j}[u, h, v]=\int d x D_{x}(\ldots)=0
$$

are trivial in the ring $\overline{\mathscr{K}}\{u, h, v\}$. Equivalently, the gradient $\varphi(x ; \lambda):=\operatorname{grad} \int d x\left(\lambda u+h+\lambda^{-1} v\right)=$ $\left(\lambda, 1, \lambda^{-1}\right)^{\top}$ satisfies the Lax equation (2.14) in the ring $\overline{\mathscr{K}}\{u, h, v\}$ and thus it should coincide with the expression (3.5). In particular, one can readily show that

$$
\varphi(x ; \lambda)=\left(\lambda, 1, \lambda^{-1}\right)^{\top} g(x ; \lambda) \exp \left[-\lambda^{3} t-\lambda^{2} x-D_{x}^{-1}\left(\lambda u+h+\lambda^{-1} v\right)\right],
$$

holds, where the scalar invertible element

$$
g(x ; \lambda):=\exp \left[-u \lambda^{-1}+\left(u^{2} / 2-h\right) \lambda^{-2}+D_{x}^{-1} \sigma_{j}[u, h, v] / \lambda^{j}\right)
$$

is a local functional from $\Lambda^{0}(\overline{\mathscr{K}}\{u, h, v\})$, giving rise to (3.2) and (3.3), which completes the proof.

As an important consequence in the gradient-holonomic integrability context $[1,36]$ of the calculations above, we can formulate the following proposition.

Proposition 3.2. The nonlinear hydrodynamic system (1.1) possesses only three $\lambda$-ordered conserved quantities that, in part, entails its Lax integrability. 
From the expression (3.2) one easily finds that the following linear differential relationships

$$
D_{x} \varphi_{j}=-\left(\lambda^{2}+u \lambda+h+v \lambda^{-1}\right) \varphi_{j}-\left[u_{x} \lambda^{-1}+\left(h_{x}-u u_{x}\right) \lambda^{-2}-r_{x}(x ; \lambda)\right] \varphi_{j}
$$

hold for $j=1,2,3$ and all $\lambda \in \mathbb{C}$, where the local functional $r(x ; \lambda) \in \Lambda^{0}(\overline{\mathscr{K}}\{u, h, v\})$ is defined as $|\lambda| \rightarrow \infty$ by means of the asymptotic expansion

$$
r(x ; \lambda) \sim \sum_{j>2} D_{x}^{-1} \sigma_{j}[u, h, v] / \lambda^{j} .
$$

The existence of the $\lambda$-ordered asymptotic solution as $|\lambda| \rightarrow \infty$ (3.5) means in the gradientholonomic integrability approach $[1,36]$, that there exists a related linear spectral problem in the differential form

$$
D_{x} f(x ; \lambda)=l[u, h, v ; \lambda] f(x ; \lambda),
$$

where, in general, a matrix mapping $l: M \times \mathbb{C} \rightarrow$ End $\Lambda^{0}(\overline{\mathscr{K}}\{u, h, v\})^{n}$ can be chosen to satisfy the natural zero-trace constraint $\operatorname{tr}(l[u, h, v ; \lambda])=0$. It is important to observe that it follows from the general theory $[9,42]$ of asymptotic solutions to linear differential equations that there is a simple relationship between the dimension $n \in \mathbb{Z}_{+}$of the matrix $l[u, h, v ; \lambda] \in \operatorname{End} \Lambda^{0}(\overline{\mathscr{K}}\{u, h, v\})^{n}$ and the number $n_{a s} \in \mathbb{Z}_{+}$of asymptotic expansions with different hierarchies of the conserved quantities for the Lax integrable nonlinear dynamical system (1.10); namely $n_{a s}+1=n$. Turning back to the characteristic system (2.14) one easily finds that it has only one nontrivial asymptotic expansion (3.2), which implies that $n=\operatorname{dim} l[u, h, v ; \lambda]=2$. Taking this into account along with

$$
\varphi_{1}(x ; \lambda)=\lambda^{-1} \varphi_{2}(x ; \lambda), \quad \varphi_{3}(x ; \lambda)=\lambda \varphi_{2}(x ; \lambda),
$$

one finds, owing to Theorem 2.1, that

$$
\varphi_{1}(x ; \lambda)=\lambda^{-1} f_{11}(x ; \lambda)^{2} e^{q(x ; \lambda)}, \varphi_{2}(x ; \lambda)=f_{11}(x ; \lambda)^{2} e^{q(x ; \lambda)}, \varphi_{3}(x ; \lambda)=\lambda f_{11}(x ; \lambda)^{2} e^{q(x ; \lambda)},
$$

for the $(1,1)$-component of the two-dimensional a priori nonlocal fundamental matrix $F(x ; \lambda) \in$ End $\Lambda^{0}\left(\overline{\mathscr{K}}\left\{u, h, v ; D_{x}^{-1} \sigma \mid 3\right\}\right)^{2}$ and some local functional $q(x ; \lambda) \in \Lambda^{0}(\overline{\mathscr{K}}\{u, h, v\})$, which can be chosen dependent only on the local functional (3.16) as $q(x ; \lambda):=-u \lambda^{-1}-\left(u^{2} / 2-h\right) \lambda^{-2}-$ $r(x ; \lambda)$. Taking into account (3.19) and (3.15), we can rewrite them as a one equation with respect to $f_{11}(x ; \lambda) \in \Lambda^{0}\left(\overline{\mathscr{K}}\left\{u, h, v ; D_{x}^{-1} \sigma \mid 3\right\}\right)$ :

$$
D_{x} f_{11}(x ; \lambda)=-\frac{1}{2}\left(\lambda^{2}+u \lambda+h+v \lambda^{-1}\right) f_{11}(x ; \lambda),
$$

which holds for all $\lambda \in \mathbb{C} \backslash\{0\}$ and $x \in \mathbb{R}$. It is now easy to find the corresponding equation for the nonlocal $(2,1)$-component $f_{21}(x ; \lambda) \in \Lambda^{0}\left(\overline{\mathscr{K}}\left\{u, h, v ; D_{x}^{-1} \sigma \mid 3\right\}\right)$ of $F(x ; \lambda)$ :

$$
D_{x} f_{21}(x ; \lambda)=\chi[u, h, v ; \lambda] f_{11}(x ; \lambda)+\frac{1}{2}\left(\lambda^{2}+u \lambda+h+v \lambda^{-1}\right) f_{11}(x ; \lambda),
$$

where the a priori local functional $\chi[u, h, v ; \lambda] \in \Lambda^{0}(\overline{\mathscr{K}}\{u, h, v\})$ can, in particular, be found from the condition that there exists a local matrix $p(l) \in \operatorname{End} \Lambda^{0}(\overline{\mathscr{K}}\{u, h, v\})^{2}$ for which the following 
two systems of the Lax-Zakharov-Shabat type equations

$$
\frac{d}{d t} f(x ; \lambda)=p(l) f(x ; \lambda)
$$

and

$$
D_{x} f(x ; \lambda)=l[u, h, v ; \lambda] f(x ; \lambda),
$$

are compatible. Here $f(x ; \lambda):=\left(f_{11}(x ; \lambda), f_{21}(x ; \lambda)\right)^{\top} \in \Lambda^{0}\left(\overline{\mathscr{K}}\left\{u, h, v ; D_{x}^{-1} \sigma \mid 3\right\}\right)$ and

$$
l[u, h, v ; \lambda]:=\left(\begin{array}{cc}
-\frac{1}{2}\left(\lambda^{2}+u \lambda+h+v \lambda^{-1}\right) & 0 \\
\chi[u, h, v ; \lambda] & \frac{1}{2}\left(\lambda^{2}+u \lambda+h+v \lambda^{-1}\right)
\end{array}\right) .
$$

Another, actually more efficient and effective, way of finding $\chi[u, h, v ; \lambda]$ consists in determining the corresponding gradient element $\varphi(x ; \lambda) \in \Lambda^{0}\left(\overline{\mathscr{K}}\left\{u, h, v ; D_{x}^{-1} \sigma \mid 3\right\}\right)^{3}$ by means of (2.27), using the linear spectral equation (3.23) and (2.23), and next checking its compatibility with the determining Nöther-Lax equation (2.14). It can be readily shown that $\chi[u, h, v ; \lambda]$ is a constant, which can be taken as $\chi[u, h, v ; \lambda]=1$, for all $\lambda \in \mathbb{C}$. Thus, the linear Lax spectral problem (3.23) for the system (1.1) is governed by the following $l[u, h, v ; \lambda]$-matrix:

$$
l[u, h, v ; \lambda]:=\left(\begin{array}{cc}
-\frac{1}{2}\left(\lambda^{2}+u \lambda+h+v \lambda^{-1}\right) & 0 \\
1 & \frac{1}{2}\left(\lambda^{2}+u \lambda+h+v \lambda^{-1}\right)
\end{array}\right) .
$$

The exact form of (3.23) makes it possible to obtain the recursion operator related to (1.1), namely $\Lambda: T^{*}(M) \times \mathbb{C} \rightarrow T^{*}(M) \times \mathbb{C}$ from (2.24) and (2.27), which should satisfy

$$
\Lambda \varphi(x ; \lambda)=\lambda \varphi(x ; \lambda)
$$

for all $\lambda \in \mathbb{C} \backslash\{0\}$ and $x \in \mathbb{R}$. The recursion operator expression:

$$
\Lambda=\left(\begin{array}{ccc}
-D_{x}^{-1} u D_{x}-D_{x}-D_{x}^{-1} h D_{x}-D_{x}^{-1} v D_{x} \\
1 & 0 & 0 \\
0 & 1 & 0
\end{array}\right)
$$

satisfying the linear operator Lax equation

$$
D_{t} \Lambda=\left[\Lambda, K^{\prime, *}\right] .
$$

on $M$ can be readily derived from (3.26) and (2.14), taking into account that the adjoint (to (3.27)) symmetry recursion operator

$$
\Phi:=\Lambda^{*}=\left(\begin{array}{ccc}
-D_{x} u D_{x}^{-1} & 1 & 0 \\
D_{x}-D_{x} h D_{x}^{-1} & 0 & 1 \\
D_{x} v D_{x}^{-1} & 0 & 0
\end{array}\right)
$$

satisfies the (adjoint to (3.28)) linear operator Lax equation

$$
D_{t} \Phi=\left[\Phi, K^{\prime}\right] .
$$

on the functional manifold $M$. 
To construct the countable hierarchy of nonlinear dynamical systems on $M$ resulting from the linear spectral problem (3.23), it suffices to calculate $[1,4,12,27,31,36]$ the degree expression

$$
\frac{d}{d t_{n}}(u, h, v)^{\top}=\Phi^{n}\left(u_{x}, v_{x}, h_{x}\right)^{\top}:=K_{n}[u, h, v]
$$

for all $n \in \mathbb{Z}_{+}$, where $t_{n} \in \mathbb{R}$ are the corresponding new evolution parameters. As a simple example of (3.31) for $n=1$, one retrieves the Burgers system (1.1)

$$
\frac{d}{d t_{1}}(u, h, v)^{\top}=\left(\begin{array}{c}
-2 u u_{x}+h_{x} \\
-(u h)_{x}+u_{x x}+v_{x} \\
-(u v)_{x}
\end{array}\right),
$$

with evolution parameter $t_{1}=t \in[0, T)$.

We next represent the countable hierarchy of nonlinear dynamical systems (3.31) in a more general alternative form. Having defined on $M$ a generating flow

$$
\frac{d}{d \tau}(u, h, v)^{\top}:=\left(D_{x} \alpha(x ; \lambda), D_{x} \beta(x ; \lambda), D_{x} \xi(x ; \lambda)\right)^{\top}
$$

with respect to an evolution parameter $\tau \in \mathbb{R}$ by means of the asymptotic expression

$$
\left(D_{x} \alpha(x ; \lambda), D_{x} \beta(x ; \lambda), D_{x} \xi(x ; \lambda)\right)^{\top} \sim \sum_{n \in \mathbb{Z}_{+}} \lambda^{-n} K_{n}[u, h, v]
$$

for the vector $\left(\alpha_{x}, \beta_{x}, \xi_{x}\right)^{\top} \in T(M) \otimes \mathbb{C}$, satisfying the important symmetry relationship

$$
\Phi\left(D_{x} \alpha(x ; \lambda), D_{x} \beta(x ; \lambda), D_{x} \xi(x ; \lambda)\right)^{\top}=\lambda\left(D_{x} \alpha(x ; \lambda), D_{x} \beta(x ; \lambda), D_{x} \xi(x ; \lambda)\right)^{\top} .
$$

This makes it possible rewrite the relationship (3.33) in the equivalent form as

$$
\frac{d}{d \tau}\left(\lambda u+h+\lambda^{-1} v\right)=D_{x}^{2} \alpha(x ; \lambda)-D_{x}\left[\left(\lambda u+h+\lambda^{-1} v\right) \alpha(x ; \lambda)\right]
$$

with local element $\alpha(x ; \lambda) \in \Lambda^{0}(\overline{\mathscr{K}}\{u, h, v\})$, owing to the expression (3.34), having the asymptotic expansion

$$
\alpha(x ; \lambda) \sim \sum_{n \in \mathbb{Z}_{+}} \lambda^{-n} \alpha_{n}[u, h, v] .
$$

as $|\lambda| \rightarrow \infty$. To rewrite each flow (3.31) from (3.36), it suffices to observe that for each $n \in \mathbb{Z}_{+}$and $\lambda \in \mathbb{C} \backslash\{0\}$

$$
\frac{d}{d t_{n}}\left(\lambda u+h+\lambda^{-1} v\right)=D_{x}^{2} \alpha_{n}(x ; \lambda)-D_{x}\left[\left(\lambda u+h+\lambda^{-1} v\right) \alpha_{n}(x ; \lambda)\right]
$$

where

$$
\alpha_{n}(x ; \lambda):=\left(\lambda^{n} \alpha(x ; \lambda)\right)_{+},
$$

that is the corresponding polynomial part of the asymptotic expansion as $|\lambda| \rightarrow \infty$ for the local functional $\lambda^{n} \alpha(x ; \lambda) \in \Lambda^{0}(\overline{\mathscr{K}}\{u, h, v\})$. 
As easily follows from the recurrent relationship (3.35), the symmetry recursion operator $\Phi$ : $T(M) \otimes \mathbb{C} \rightarrow T(M) \otimes \mathbb{C}$ satisfies the differential relationships

$$
\frac{d}{d t_{n}} \Phi=\left[K^{\prime}, \Phi\right]
$$

for all $n \in \mathbb{Z}_{+}$. This implies that the whole countable hierarchy of (3.31) are mutually commuting.

Remark 3.1. All the analysis above was performed for the case when the local functional $\chi[u, h, v ; \lambda] \in \Lambda^{0}(\overline{\mathscr{K}}\{u, h, v\})$ in the $l$-operator (3.24) was constant owing to the compatibility between (2.27) and (2.14). If this local functional is assumed to be nonconstant, it is easy to see that (3.24) is significantly modified, giving rise to a different symmetry recursion operator expression, which generates another countable hierarchy of nonlinear dynamical systems on $M$.

Recall now that for the linear Lax spectral problem (3.23) there should be associated evolution equations (3.22) for the vector function $f=\left(f_{11}, f_{21}\right)^{\top} \in \Lambda^{0}\left(\overline{\mathscr{K}}\left\{u, h, v ; D_{x}^{-1} \sigma \mid 3\right\}\right)^{2}$ of the form

$$
\frac{d}{d t_{n}} f(x ; \lambda)=p_{n}(l) f(x ; \lambda),
$$

governed by matrices $p_{n}(l) \in E n d \Lambda^{0}\left(\overline{\mathscr{K}}\left\{u, h, v ; D_{x}^{-1} \sigma \mid 3\right\}\right)^{2}, n \in \mathbb{Z}_{+}$, which are yet to be determined. To construct these matrices, we shall use (3.38) and observe that the following linear evolution equations are compatible with that of (3.20) for all $n \in \mathbb{Z}_{+}$.Similarly, we have

$$
\frac{d}{d t_{n}} f_{21}(x ; \lambda)=\eta_{n}[u, h, v ; \lambda] f_{11}(x ; \lambda)-\frac{1}{2}\left[\left(\lambda^{2}+u \lambda+h+v \lambda^{-1}\right) \alpha_{n}(x ; \lambda)-D_{x} \alpha_{n}(x ; \lambda)\right] f_{21}(x ; \lambda),
$$

where the local functionals $\eta_{n}[u, h, v ; \lambda] \in \Lambda^{0}\left(\overline{\mathscr{K}}\left\{u, h, v ; D_{x}^{-1} \sigma \mid 3\right\}\right), n \in \mathbb{Z}_{+}$, are to be determined from the corresponding compatibility condition of the equations (3.42) with vector component equation (3.21). Simple calculations yield

$$
D_{x} \eta_{n}(x ; \lambda)-\left(\lambda^{2}+u \lambda+h+v \lambda^{-1}\right) \eta_{n}(x ; \lambda)=\left(\lambda^{2}+u \lambda+h+v \lambda^{-1}\right) \alpha_{n}(x ; \lambda)-D_{x} \alpha_{n}(x ; \lambda),
$$

which holds for all $\lambda \in \mathbb{C} \backslash\{0\}$ and $n \in \mathbb{Z}_{+}$, and make it possible to find that

$$
\eta_{n}(x ; \lambda)=-\alpha_{n}(x ; \lambda)
$$

for all $n \in \mathbb{Z}_{+}$. Thus, the vector function $f=\left(f_{11}, f_{21}\right)^{\top} \in \Lambda^{0}\left(\overline{\mathscr{K}}\left\{u, h, v ; D_{x}^{-1} \sigma \mid 3\right\}\right)^{2}$ satisfies the evolution equations (3.41) with the governing matrix expressions

$$
p_{n}(l)=\left(\begin{array}{cc}
\frac{1}{2}\left[\left(\lambda^{2}+u \lambda+h+v \lambda^{-1}\right) \alpha_{n}(x ; \lambda)\right. & 0 \\
\left.-D_{x} \alpha_{n}(x ; \lambda)\right] & \frac{1}{2}\left[D_{x} \alpha_{n}(x ; \lambda)-\right. \\
-\alpha_{n}(x ; \lambda) & \left.-\left(\lambda^{2}+u \lambda+h+v \lambda^{-1}\right) \alpha_{n}(x ; \lambda)\right]
\end{array}\right)
$$

for all $n \in \mathbb{Z}_{+}$and $\lambda \in \mathbb{C} \backslash\{0\}$.

Summing up the results obtained above, one has the following result.

Proposition 3.3. The system (1.1) is a Lax integrable flow on the functional manifold M, whose linear spectral problems are given by (3.22) and (3.23), governed by the matrices (3.45) at $n=1$ and (3.25), respectively. The countable hierarchy of nonlinear dynamical systems on $M$ related to the linear spectral problem (3.23) is given by the mutually commuting evolution flows (3.31), 
whose compatible temporal linear spectral problems (3.41) are governed by (3.45). Moreover, all of these systems possess only three nontrivial local conserved quantities $\gamma_{-1}=\int d x u, \gamma_{0}=\int d x h$ and $\gamma_{1}=\int d x u v$.

It should be mentioned here that the interesting question of existence of a countable hierarchy of nonlocal conserved quantities for the nonlinear system (1.1) remains to be answered. It is also reasonable to consider describing all dynamical systems that can be obtained from the linear spectral problem (3.23) with the governing matrix (3.24), in which the local functional $\chi[u, h, v ; \lambda]$ is taken to be non-constant. In particular, it can be assumed that $\chi[u, h, v ; \lambda]=\sum_{j=0}^{p} \lambda^{j} w_{j}, p \in \mathbb{Z}_{+}$, for an additional phase space component $w \in W \subset C^{\infty}\left(\mathbb{R} ; \mathbb{R}^{m+1}\right)$ of the extended functional manifold $\tilde{M}:=$ $M \times W$, on which the related nonlinear dynamical systems are defined. Other generalizations of (3.23), governed by (3.24), will be considered in the next subsection.

\subsection{Integrability analysis of the dispersive flow (1.2)}

The system (1.2) can also be analyzed by means of the gradient-holonomic approach together with related differential-algebraic techniques, which was successfully applied above to the nonlinear Burgers type system (1.1). First we need to construct asymptotic (as $|\lambda| \rightarrow \infty$ ) solutions to the linear Nöther-Lax equation (2.14) with the (calculated) governing operator

$$
K^{\prime, *}[u, h, v]=\left(\begin{array}{ccc}
2 u D_{x}+D_{x}^{2} & h D_{x} & v D_{x} \\
-D_{x} & u D_{x} & 0 \\
0 & -D_{x} & u D_{x}
\end{array}\right),
$$

acting in the space $\Lambda^{0}\left(\overline{\mathscr{K}}\left\{u, h, v ; D_{x}^{-1} \sigma \mid N\right\}\right)^{3}$ for some still not defined $N \in \mathbb{Z}_{+}$. The corresponding basic equations are

$$
\begin{aligned}
d \varphi_{1} / d t+2 u \varphi_{1, x}+\varphi_{1, x x}+h \varphi_{2, x}+v \varphi_{3, x} & =0, \\
d \varphi_{2} / d t+u \varphi_{2, x}-\varphi_{1, x} & =0, \\
d \varphi_{3} / d t-\varphi_{2, x}+u \varphi_{3, x} & =0,
\end{aligned}
$$

whose asymptotic solution as $|\lambda| \rightarrow \infty$ have following form:

$$
\begin{aligned}
& \varphi_{1}(x ; \lambda)=\exp \left[-\lambda^{2} t+\lambda x+D_{x}^{-1} \sigma(x ; \lambda)\right], \\
& \varphi_{2}(x ; \lambda)=a(x ; \lambda) \varphi_{1}(x ; \lambda), \varphi_{3}(x ; \lambda)=b(x ; \lambda) \varphi_{1}(x ; \lambda),
\end{aligned}
$$

where

$$
\sigma(x ; \lambda) \sim \sum_{j \in \mathbb{Z}_{+}} \lambda^{-j} \sigma_{j}[u, h, v], a(x ; \lambda) \sim \sum_{j \in \mathbb{Z}_{+}} \lambda^{-j} a_{j}[u, h, v], b(x ; \lambda) \sim \sum_{j \in \mathbb{Z}_{+}} \lambda^{-j} b_{j}[u, h, v],
$$

for some local functionals $\sigma_{j}[u, h, v], a_{j}[u, h, v]$ and $b_{j}[u, h, v] \in \Lambda^{0}(\overline{\mathscr{K}}\{u, h, v\}), j \in \mathbb{Z}_{+}$. The representation (3.48) assumes, in particular, that

$$
\gamma(\lambda):=\int d x \sigma(x ; \lambda)
$$


is a generating functional of conserved quantities for (1.2). By substituting (3.48) into (3.47), one readily obtains

$$
\begin{gathered}
D_{t} a_{j}-a_{j+2}+\sum_{k \in \mathbb{Z}_{+}} a_{j-k} D_{x}^{-1} d \sigma_{k} / d t+u a_{j, x}+u a_{j+1}+ \\
+u \sum_{k \in \mathbb{Z}_{+}} a_{j-k} \sigma_{k}-\delta_{j,-1}-\sigma_{j}=0, \\
d b_{j} / d t-b_{j+2}+\sum_{k \in \mathbb{Z}_{+}} b_{j-k} D_{x}^{-1} d \sigma_{k} / d t-a_{j, x}-a_{j+1}- \\
-\sum_{k \in \mathbb{Z}_{+}} a_{j-k} \sigma_{k}+u b_{j, x}+u b_{j+1}+u \sum_{k \in \mathbb{Z}_{+}} b_{j-k} \sigma_{k}=0, \\
D_{x}^{-1} d \sigma_{j} / d t+2 u \delta_{j,-1}+2 u \sigma_{j}+2 \sigma_{j+1}+\sum_{k \in \mathbb{Z}_{+}} \sigma_{j-k} \sigma_{k}+\sigma_{j, x}++h a_{j, x}+ \\
+h a_{j+1}+h \sum_{k \in \mathbb{Z}_{+}} a_{j-k} \sigma_{k}+v b_{j, x}+v b_{j+1}+v \sum_{k \in \mathbb{Z}_{+}} b_{j-k} \sigma_{k}=0
\end{gathered}
$$

for all $j \in \mathbb{Z}_{+} \cup\{-2,-1\}$. Whence, the coefficients are easily found to be

$$
\begin{aligned}
& \sigma_{0}=-u, \sigma_{1}=h+u_{x}, \sigma_{2}=-v-D_{x}\left(u_{x}+h-u^{2}\right), \ldots, \\
& \sigma_{j}=D_{x} r_{j+3}[u, h, v], \ldots ; \\
& a_{0}=0, a_{1}=-1, a_{2}=0, \ldots, a_{j+3}=0, \ldots \\
& b_{0}=0, b_{1}=0, b_{2}=1, \ldots, b_{j+3}=0, \ldots
\end{aligned}
$$

which hold for all $j \in \mathbb{Z}_{+}$. It follows from (3.48) taken together with (3.49) and (3.52), that

$$
\varphi_{2}(x ; \lambda)=-\lambda^{-1} \varphi_{1}(x ; \lambda), \varphi_{3}(x ; \lambda)=\lambda^{-2} \varphi_{1}(x ; \lambda)
$$

hold for all $\lambda \in \mathbb{C} \backslash\{0\}$ and $x \in \mathbb{R}$. Having recalled now the results from Subsection 3.1 concerning the application of the gradient-holonomic approach $[1,35,36]$ to the solutions of the determining Nöther-Lax equation (2.14), one can easily infer the existence a nontrivial hierarchy of conserved quantities containing only the three local conserved quantities

$$
\gamma_{0}=\int d x u, \quad \gamma_{1}=\int d x h, \quad \gamma_{2}=\int d x v
$$

This entails the existence of two-dimensional linear Lax type spectral problems (2.16), whose squared $(1,1)$-component of the fundamental matrix $F(x ; \lambda) \in$ End $\Lambda^{0}\left(\overline{\mathscr{K}}\left\{u, h, v ; D_{x}^{-1} \sigma \mid 3\right\}\right)^{2}$ satisfies, owing to (3.53), the functional relationships

$$
\varphi_{1}(x ; \lambda)=f_{11}(x ; \lambda)^{2} e^{q}, \varphi_{2}(x ; \lambda)=-\lambda^{-1} f_{11}(x ; \lambda)^{2} e^{q}, \varphi_{3}(x ; \lambda)=-\lambda^{-2} f_{11}(x ; \lambda)^{2} e^{q},
$$

where

$$
q=q(x ; \lambda) \sim \lambda^{-1} u-\lambda^{-2}\left(u_{x} h-u^{2}\right)-\sum_{j \in \mathbb{Z}_{+}} \lambda^{-3-j} D_{x}^{-1} \sigma_{j}[u, h, v],
$$

as $|\lambda| \rightarrow \infty$ is a local functional from $\Lambda^{0}(\overline{\mathscr{K}}\{u, h, v\})$. Now it is easy to obtain from (3.48) and (3.55) an equivalent system of the linear Lax spectral equations of the form

$$
\begin{aligned}
& D_{x} f_{11}=\frac{1}{2}\left(\lambda-u+\lambda^{-1} h-\lambda^{-2} v\right) f_{11}, \\
& D_{x} f_{21}=\chi[u, h, v ; \lambda] f_{11}-\frac{1}{2}\left(\lambda-u+\lambda^{-1} h-\lambda^{-2} v\right) f_{21}
\end{aligned}
$$

for a vector $f=\left(f_{11}, f_{21}\right)^{\top} \in \Lambda^{0}\left(\overline{\mathscr{K}}\left\{u, h, v ; D_{x}^{-1} \sigma \mid 3\right\}\right)^{2}$, where $\lambda \in \mathbb{C} \backslash\{0\}$ and the local functional $\chi[u, h, v ; \lambda] \in \Lambda^{0}(\overline{\mathscr{K}}\{u, h, v\})$ is determined from the compatibility of (3.57) with (2.14). Simple calculations show that $\chi[u, h, v ; \lambda]$ is constant, which we choose for brevity as $\chi[u, h, v ; \lambda]=1$ for 
all $\lambda \in \mathbb{C} \backslash\{0\}$ and $(u, h, v)^{\top} \in M$. Thus, the Lax spectral equations (3.57) for the system (1.2) can be rewritten in the matrix form (2.16), where the matrix $l[u, h, v ; \lambda] \in E n d \Lambda^{0}\left(\overline{\mathscr{K}}\left\{u, h, v ; D_{x}^{-1} \sigma \mid 3\right\}\right)^{2}$ is

$$
l[u, h, v ; \lambda]:=\left(\begin{array}{cc}
\frac{1}{2}\left(\lambda-u+\lambda^{-1} h-\lambda^{-2} v\right) & 0 \\
1 & \left.\frac{1}{2}\left(-\lambda+u-\lambda^{-1} h+\lambda^{-2} v\right)\right)
\end{array}\right)
$$

and is defined for all $\lambda \in \mathbb{C} \backslash\{0\}$ and $(u, h, v)^{\top} \in M$. Then, using (2.16) and (2.24) one can readily construct the related recursion operator

$$
\Lambda=\left(\begin{array}{ccc}
D_{x}+D_{x}^{-1} u D_{x} & D_{x}^{-1} h D_{x} D_{x}^{-1} v D_{x} \\
-1 & 0 & 0 \\
0 & -1 & 0
\end{array}\right)
$$

which implicitly satisfies the recurrent relationship

$$
\Lambda \varphi(x ; \lambda)=-\lambda \varphi(x ; \lambda)
$$

for the vector $\varphi(x ; \lambda) \in \Lambda^{0}\left(\overline{\mathscr{K}}\left\{u, h, v ; D_{x}^{-1} \sigma \mid 3\right\}\right)^{3}$ satisfying (2.14). The symmetry recursion operator adjoint to (3.59) is

$$
\Phi:=\Lambda^{*}=\left(\begin{array}{ccc}
-D_{x}+D_{x} u D_{x}^{-1} & -1 & 0 \\
D_{x} h D_{x}^{-1} & 0 & -1 \\
D_{x} v D_{x}^{-1} & 0 & 0
\end{array}\right),
$$

which acts on $T(M) \otimes \mathbb{C}$, and allows to generate a countable hierarchy of mutually commuting nonlinear dynamical systems on $M$ via the degree iteration

$$
\frac{d}{d t_{n}}(u, h, v)^{\top}:=\Phi^{n}\left(u_{x}, h_{x}, v_{x}\right)^{\top}
$$

for any $n \in \mathbb{Z}_{+}$, whose first flow coincides with the nonlinear dynamical system (1.2)

$$
\frac{d}{d t}(u, h, v)^{\top}=\Phi\left(u_{x}, h_{x}, v_{x}\right)^{\top}=\left(\begin{array}{c}
u_{x x}+h_{x}-2 u u_{x} \\
-(u h)_{x} \\
-(u v)_{x}
\end{array}\right) .
$$

If we now define a symmetry generating vector function $\left(D_{x} \alpha, D_{x} \beta, D_{x} \xi\right)^{\top} \in T(M) \otimes \mathbb{C}$ as

$$
\left(D_{x} \alpha, D_{x} \beta, D_{x} \xi\right)^{\top}:=\sum_{j \in \mathbb{Z}_{+}} \lambda^{-j} \Phi^{j}\left(u_{x}, h_{x}, v_{x}\right)^{\top},
$$

satisfying the linear recurrence relationship

$$
\Phi\left(D_{x} \alpha, D_{x} \beta, D_{x} \xi\right)^{\top}:=\lambda\left(D_{x} \alpha, D_{x} \beta, D_{x} \xi\right)^{\top}
$$

as $|\lambda| \rightarrow \infty$, the hierarchy (3.63) can be rewritten as

$$
\frac{d}{d t_{n}}\left(\lambda-u+\lambda^{-1} h-\lambda^{-2} v\right)=D_{x}\left[D_{x} \alpha_{n}(x ; \lambda)+\left(\lambda-u+\lambda^{-1} h-\lambda^{-2} v\right) \alpha_{n}(x ; \lambda)\right] .
$$

Here,

$$
\alpha_{n}:=\left(\lambda^{n} \alpha(x ; \lambda)_{+}\right.
$$


for any $n \in \mathbb{Z}_{+}$and the coefficients of the asymptotic (as $\left.|\lambda| \rightarrow \infty\right)$ series

$$
\alpha(x ; \lambda) \sim \sum_{j \in \mathbb{Z}_{+}} \lambda^{-j} \alpha_{j}[u, h, v]
$$

should be determined from the differential relationship

$$
D_{x}^{2} \alpha(x ; \lambda)+D_{x}\left[\left(\lambda-u+\lambda^{-1} h-\lambda^{-2} v\right) \alpha(x ; \lambda)\right]=0 .
$$

As in, one can obtain the compatible evolution equations

$$
\frac{d}{d t_{n}} f(x ; \lambda)=p_{n}(l) f(x ; \lambda),
$$

for the vector function $f(x ; \lambda) \in \Lambda^{0}\left(\overline{\mathscr{K}}\left\{u, h, v ; D_{x}^{-1} \sigma \mid 3\right\}\right)^{2}$, where matrices $p_{n}(l) \in$ $\operatorname{End} \Lambda^{0}\left(\overline{\mathscr{K}}\left\{u, h, v ; D_{x}^{-1} \sigma \mid 3\right\}\right)^{2}, n \in \mathbb{Z}_{+}$, are

$$
p_{n}(l)=\left(\begin{array}{cc}
\frac{1}{2}\left[D_{x} \alpha_{n}(x ; \lambda)+\right. & 0 \\
\left.+\left(\lambda-u+\lambda^{-1} h-\lambda^{-2} v\right) \alpha_{n}(x ; \lambda)\right] & \\
-\alpha_{n}(x ; \lambda) & +\frac{1}{2}\left[D_{x} \alpha_{n}(x ; \lambda)+\right. \\
& \left.+\left(\lambda-u+\lambda^{-1} h-\lambda^{-2} v\right) \alpha_{n}(x ; \lambda)\right]
\end{array}\right),
$$

which can be easily obtained from the determining compatibility matrix conditions

$$
\frac{d}{d t_{n}} l=\left[p_{n}(l), l\right]+D_{x} p_{n}(l)
$$

for all $\lambda \in \mathbb{C} \backslash\{0\}$ and $n \in \mathbb{Z}_{+}$. For $n=1$, the evolution equation (2.17) is governed by the matrix expression

$$
p(l)=\left(\begin{array}{cc}
\frac{1}{2}\left[\left(\lambda-u+\lambda^{-1} h-\lambda^{-2} v\right) \times\right. & \\
\left.\times(\lambda+u)-u_{x}\right] & 0 \\
\lambda+u & \left.\times\left(\lambda-u+\lambda^{-1} h-\lambda^{-2} v\right)\right]
\end{array}\right) .
$$

The following result follows directly from the above considerations.

Proposition 3.4. The Burgers system (1.2) is integrable by means of the Lax linear spectral problems (2.16) and (2.17), with corresponding two-dimensional matrix expressions (3.58) and (3.73). Moreover, it possesses a countable hierarchy of mutually commuting nonlinear dynamical systems (3.62), generated by (3.61), which allows no factorization by means of any compatible pair of Poisson operators on $M$.

It is worth mentioning that the existence of a countable hierarchy of nonlocal conserved quantities for the Burgers system (1.2) has yet to be completely verified, even though for $h=0=v$ it does prove to possess [37] such a nonlocal hierarchy, which is generated by the corresponding recursion operator, whose squared form can be factorized by means of compatible Poisson structures.

Nonetheless, as shown in [37], if one can prove that (1.2) has another infinite hierarchy of nonlocal conservation laws, then some portion of the symmetry recursion operator found in (3.61) will already be factorized by means of the constructed Poisson structures - but this is still an open problem. 


\subsection{The integrable Kaup-Broer generalization of the Burgers systems}

The linear Lax spectral problem (3.57), defined by the matrix (3.58), can be naturally generalized in the form

$$
D_{x} f(x ; \lambda)=l[\mathbf{u}, v ; \lambda] f(x ; \lambda)
$$

with the square matrix

$$
l[u, v ; \lambda]:=\left(\begin{array}{cc}
\frac{k}{2}\left(\lambda^{n+1}-\sum_{j=-m}^{n} u_{j} \lambda^{j}\right) & -\frac{k}{2}\left(\sum_{j=0}^{n} v_{j} \lambda^{j}\right) \\
\sum_{j=0}^{n} c_{j} \lambda^{j} & -\frac{k}{2}\left(\lambda^{n+1}-\sum_{j=-m}^{n} u_{j} \lambda^{j}\right)
\end{array}\right),
$$

where $(u, v)^{\top}:=\left(u_{-m}, u_{2}, \ldots, u_{n}, v_{0}, v_{1}, \ldots, v_{n}\right)^{\top} \in M \subset C^{\infty}\left(\mathbb{R} ; \mathbb{R}^{2(n+1)+m}\right), c_{j} \in \mathbb{R}, j=0, \ldots n$, are constants and $\lambda \in \mathbb{C} \backslash\{0\}$ is a spectral parameter. Using (3.74) in the Sobolev space $W_{2}^{(1)}\left(\mathbb{R} ; \mathbb{C}^{2}\right)$, one can readily obtain, along the lines of the work in the preceding sections, the integrable biHamiltonian dynamical systems

$$
d(\mathbf{u}, \mathbf{v})^{\top} / d t_{j}=K_{j}[\mathbf{u}, \mathbf{v}]
$$

on $M$ with respect to the countable hierarchy of evolution parameters $t_{j} \in \mathbb{R}, j \in \mathbb{Z}_{+}$. We hope to present a detailed analysis in a forthcoming paper.

In the special case $n=0=m, c_{0}=1$ and $k=1 / 2,(3.75)$ gives rise to the linear Lax spectral problem

$$
D_{x} f(x ; \lambda)=l[u, h ; \lambda] f(x ; \lambda)
$$

in $W_{2}^{(1)}\left(\mathbb{R} ; \mathbb{C}^{2}\right)$, governed by the matrix operator

$$
l[u, v ; \lambda]=\left(\begin{array}{cc}
\frac{1}{2}(\lambda-u) & -\frac{1}{4} h \\
1 & -\frac{1}{2}(\lambda-u)
\end{array}\right)
$$

of the well known Kaup-Broer-Kupershmidt system (1.5), whose integrability was studied in detail in $[5,8,19,23]$. The corresponding Lax matrix operator with respect to $t \in \mathbb{R}$ is

$$
p(l):=\left(\begin{array}{cc}
\frac{1}{8}\left[\left(u^{2}-\lambda^{2}\right)-2 u_{x}\right] & \frac{1}{4} h_{x}+\frac{1}{8} h(u+\lambda) \\
\frac{1}{2}(u+\lambda) & -\frac{1}{8}\left[\left(u^{2}-\lambda^{2}\right)-2 u_{x}\right]
\end{array}\right)
$$

and it satisfies the commutator Lax-Zakharov-Shabat matrix condition (3.72) on a suitably defined symplectic functional manifold $M \subset C^{\infty}\left(\mathbb{R} ; \mathbb{R}^{2}\right)$, endowed with a compatible pair of Poisson operators $\vartheta, \eta: T^{*}(M) \rightarrow T(M)$ :

$$
\vartheta=\left(\begin{array}{cc}
0 & D_{x} \\
D_{x} & 0
\end{array}\right), \quad \eta=\left(\begin{array}{cc}
2 D_{x} & 2 D_{x}^{2}+D_{x} u \\
2 D_{x}^{2}-u D_{x} & v D_{x}+D_{x} v
\end{array}\right) .
$$

Remark 3.2. It should be mentioned that when $h=0$ the Kaup-Broer-Kupershmidt nonlinear dynamical system $(1.5)$ reduces $[37,45]$ to a bi-Hamiltonian Burgers dynamical system on a suitably reduced functional manifold $\tilde{M}$ with respect to the following compatible Poisson operators:

$$
\tilde{\vartheta}=\partial \exp \left(\partial^{-1} u\right) \partial \exp \left(\partial^{-1} u\right) \partial, \quad \tilde{\eta}=\partial \exp \left(\partial^{-1} u\right) \partial^{3} \exp \left(\partial^{-1} u\right) \partial .
$$

Here we mean by a suitably reduced manifold, one constructed by the Novikov-Bogoyavlensky reduction scheme $[1,29,36]$, which yields an invariant submanifold $\tilde{M}$ of $M$ with the desired 
property. In particular, the corresponding reduction of the operators (3.81) to $\tilde{M}$ yields the biHamiltonian structure for the Burgers equation. The associated symplectic structures for Burgers equation restricted to $\tilde{M}$ can be obtained using the well-known Gelfand-Dickey differential method. An equivalent of this result was first obtained by Taflin [44] in 1981. In this regard, it should be noted that the bi-Hamiltonian representation of Burgers equation can also be obtained from the real reduction of the analogous structure for its complex form, $u_{t}=-i u_{x}-2 u u_{x}$, discovered by Brushi \& Ragnisco [6].

Similarly, if we set $n=1=m, c_{0}=0, c_{1}=1, u_{0}:=u, u_{1}:=0, v_{0}:=0, v_{1}:=h$ and $k=1$ in (3.75), one obtains the linear Lax type spectral problem

$$
D_{x} f(x ; \lambda)=l[u, h ; \lambda] f(x ; \lambda), \quad l[u, h ; \lambda]:=\left(\begin{array}{cc}
\frac{1}{2}\left(\lambda^{2}-u\right)-\lambda h \\
\lambda & 0
\end{array}\right)
$$

in $W_{2}^{(1)}\left(\mathbb{R} ; \mathbb{C}^{2}\right)$ for the following dark integrable bi-Hamiltonian nonlinear hydrodynamic system

$$
\begin{aligned}
& u_{t}=-2 u_{x x}+8 u u_{x}+8(h u)_{x}+4 h h_{x}-4 h_{x x}, \\
& h_{t}=2 h_{x x}+4 u h_{x}+4 h h_{x}
\end{aligned}
$$

on $M \subset C^{\infty}\left(\mathbb{R} ; \mathbb{R}^{2}\right)$.

An important generalization of the linear Lax spectral problem (3.74) comes from the interesting work [38], and has the form

$$
D_{x} f(x ; \lambda)=l[\mathbf{u}, v, h ; \lambda] f(x ; \lambda),
$$

which is governed by the square matrix

$$
l[u, v, h ; \lambda]:=\left(\begin{array}{cc}
\frac{h}{2}\left(\lambda^{n+1}-\sum_{j=-m}^{n} u_{j} \lambda^{j}\right) & -\frac{h}{2}\left(\sum_{j=0}^{n} v_{j} \lambda^{j}\right) \\
\sum_{j=0}^{n} c_{j} \lambda^{j} & -\frac{h}{2}\left(\lambda^{n+1}-\sum_{j=-m}^{n} u_{j} \lambda^{j}\right)
\end{array}\right),
$$

where $(u, v, h)^{\top}:=\left(u_{-m}, u_{2}, \ldots, u_{n}, v_{0}, v_{1}, \ldots, v_{n}, h\right)^{\top} \in M \subset C^{\infty}\left(\mathbb{R} ; \mathbb{R}^{2 n+m+3}\right), c \in \mathbb{R}$ is a constant and $\lambda \in \mathbb{C} \backslash\{0\}$ is a spectral parameter. In the special case $n=0=m, u_{0}:=u$, and $c_{0}=0=v_{0}$, the matrix operator (3.85) gives rise to the following compatible pair of the linear matrix Lax spectral problems; namely,

$$
D_{x} f(x ; \lambda)=l[u, h ; \lambda] f(x ; \lambda), l[u, h ; \lambda]=\left(\begin{array}{cc}
\frac{h}{2}(\lambda-u) & 0 \\
0 & -\frac{h}{2}(\lambda-u)
\end{array}\right)
$$

and

$$
\frac{d}{d t} f(x ; \lambda)=p(l) f(x ; \lambda), p(l)=\left(\begin{array}{cc}
\frac{u h}{2}(\lambda-u)-\frac{h}{2} h_{x x}+\frac{1}{4} h_{x}^{2} & 0 \\
0 & \frac{h}{2} h_{x x}-\frac{u h}{2}(\lambda-u)-\frac{1}{4} h_{x}^{2}
\end{array}\right),
$$

which are equivalent to the following noteworthy dark nonlinear Hamiltonian hydrodynamic system

$$
\begin{aligned}
& u_{t}=-u u_{x}+h_{x x x}, \\
& h_{t}=-u_{x} h-u h_{x}
\end{aligned}
$$

with respect to the Poisson structure

$$
\vartheta=\left(\begin{array}{cc}
0 & D_{x} \\
D_{x} & 0
\end{array}\right)
$$


on $M \subset C^{\infty}\left(\mathbb{R} ; \mathbb{R}^{2}\right)$.

Another physically interesting case is obtained for $k=n=1, m=0, c_{0}=1, c_{1}=0, v_{0}=v_{1}=0$, $u_{0}=-h u$ and $u_{1}=h$ in (3.85). The corresponding linear Lax spectral problems

$$
D_{x} f(x ; \lambda)=l[u, h ; \lambda] f(x ; \lambda), l[u, h ; \lambda]=\left(\begin{array}{cc}
\frac{h}{2}(\lambda-u)+\frac{\lambda^{2}}{2} & 0 \\
1 & -\frac{h}{2}(\lambda-u)-\frac{\lambda^{2}}{2}
\end{array}\right)
$$

enable the construction of a new dark integrable bi-Hamiltonian system

$$
\begin{aligned}
& u_{t}=-u_{x}(u-h)-h^{-1}\left(u_{x x}+h_{x} u^{2}+h_{x x}\right), \\
& h_{t}=u_{x} h+u h_{x}+2 h h_{x},
\end{aligned}
$$

on a suitably defined Poissonian functional manifold $M \subset C^{\infty}\left(\mathbb{R} ; \mathbb{R}^{2}\right)$.

\section{Concluding Remarks}

Using a combined gradient-holonomic-differential-algebraic approach $[1,32,33,36,37]$ to test the Lax integrability of nonlinear dynamical systems, we showed that the three-component Burgers systems (1.1) and (1.2) possess 2-dimensional matrix Lax representations and corresponding symmetry recursion operators - which do not allow the usual bi-Poisoning factorization. In particular, (1.2) does not have a related countable hierarchy of, either local or non- local, conserved quantities. The problem of constructing a generalized bi-Poisoning factorization of a suitable power of a recursion operator, such as in [37], is left for a future analysis, and so is an investigation of integrable countable hierarchies of "dark"systems related to the Lax spectral problems (3.74) and (3.84). Thus, gradient-holonomic and differential-algebraic tools combined in the context of symplectic geometry can serve as a relatively simple and effective tool for analyzing the Lax integrability of a wide class of nonlinear polynomial dynamical systems. Moreover, as recently shown in [34], this approach also appears to be useful for nonlocal polynomial dynamical systems.

\section{Acknowledgements}

D.B. thanks the NSF (Grant CMMI-1029809) for support, and A.P. cordially thanks Prof. J. Cieślińskiemu (Białystok University, Poland) and Prof. I. Mykytyuk (Pedagogical University of Krakow, Poland) for useful discussions. Thanks are due also to the reviewers for their constructive criticism.

\section{References}

[1] D. Blackmore, A.K. Prykarpatsky and V.H. Samoylenko, Nonlinear Dynamical Systems of Mathematical Physics ( World Scientific, NJ, USA, 2011).

[2] D. Blackmore, Y.A Prykarpatsky, N.N. Bogolubov (Jr.) and A.K. Prykarpatski, Integrability of and differential-algebraic structures for spatially 1D hydrodynamical systems of Riemann type, Chaos, Solitons \& Fractals 59 (2014) 59-81.

[3] D. Blackmore, A.K. Prykarpatsky, K. Soltanov and E. Özçağ, The Lax integrability property of a two-component hierarchy of the Burgers type dynamical systems within asymptotic and differentialalgebraic approaches. arXiv:1309.5267v5 [nlin.SI] 20 Dec 2013.

[4] M. Błaszak, Bi-Hamiltonian Dynamical Systems (Springer,NY, 1998).

[5] N.N. Bogolubov (Jr.) and A.K. Prykarpatsky, Complete integrability of the nonlinear Ito and BenneyKaup systems: gradient algorithm and Lax representation, Theoretical and Mathematical Physics, 67 
(1986) 586-596 (Translated from Teoreticheskaya i Matematicheskaya Fizika 67 (1986) 410-425. Original article submitted October 17, 1985; the main results of the paper were presented at the All-Union Working Symposium Theory of Solitons and Applications, Dubna, Sept., 1985).

[6] M. Brushi and O. Ragnisco, The hamiltonian structure of a complex version of the Burgers equation, $J$. Math. Phys. 26 (1985) 943-945.

[7] F. Calogero and A. Degasperis, Spectral Transform and Solitons (Elsevier, Amsterdam, 2011).

[8] Chen Tao, Zhu Li-Li and Zhang Lei, The generalized Broer-Kaup-Kupershmidt system and its Hamiltonian extension, Appl. Math. Sci. 5 (2011) 3767 - 3780.

[9] E.A. Coddington and N. Levinson, Theory of Differential Equations (McGraw-Hill, NY, 1955).

[10] L.D. Faddeev and L.A. Takhtadjan, Hamiltonian Methods in the Theory of Solitons (Springer, New York, Berlin, 2000).

[11] M.V. Foursov, On integrable coupled Burgers-type equation, Phys. Lett.A 272 (2000) 57-64.

[12] A. Fokas and B. Fuchssteiner, Bäcklund transformations hereditary symmetries, Physica D 4 (1981) 47-66.

[13] I.M. Gelfand and L.A. Dickey, Integrable nonlinear equations and Liouville theorem, Func. Anal. Appl. 13 (1979) 8-20.

[14] I.M. Gelfand and L.A. Dickey, The calculus of jets and nonlinear Hamiltonian systems, Func. Anal. Appl. 12 (1978) 81-94.

[15] I.M. Gelfand and L.A. Dickey, A Lie algebra structure in a formal variational calculation, Func. Anal. Appl. 10 (1976) 16-22.

[16] I.M. Gelfand and L.A. Dickey, The resolvent and Hamiltonian systems, Func. Anal. Appl. 11 (1977) 93-105.

[17] I.M. Gelfand, Yu.I. Manin and M.A. Shubin, Poisson brackets and the kernel of the variational derivative in the formal calculus of variations, Func. Anal. Appl. 10 (1976) 274-278.

[18] C. Godbillon, Géométrie Différentielle et Mécanique Analytique (Hermann, Paris, 1969).

[19] O.E. Hentosh and M.M. Prytula, Hamiltonian invariant reduction of the Burgers equation, Visnyk Lviv University, Ser. Applied Mathematics and Computer Science, N1 (1999), 76-81.

[20] N.H. Ibragimov and A.B. Shabat, Infinite Lie-Backlund algebras, Func. Anal. Appl. 14 (1980) 313-315.

[21] I. Kaplanski, Introduction to Differential Algebra (NYAS, 1957).

[22] B.A. Kupershmidt, Dark equations, J. Nonlin. Math. Phys. 8 (2001) 363-445.

[23] B.A. Kupershmidt, Mathematics of dispersive water waves, Commun. Math. Phys. 99 (1985) 51-73.

[24] P.D. Lax, Almost Periodic Solutions of the KdV Equation. Source: SIAM Review, Vol. 18, No. 3 (Jul., 1976) 351-375.

[25] J.L. Lions, Quelgues Methodes de Resolution des Problemes aux Limites Non lineaires (Dunod, Paris, 1969).

[26] W.X. Ma, A hierarchy of coupled Burgers systems possessing a hereditary structure, J. Phys. A: Math. Gen. 26 (1993) L1169-74.

[27] F. Magri, A simple model of the integrable Hamiltonian equation, J. Math. Phys. 19 (1978) 1156-1162.

[28] A. Newell, Solitons in Mathematics and Physics (SIAM, Providence, 1985).

[29] S.P. Novikov (Editor), Theory of Solitons: the Inverse Scattering Method (Springer, New York, 1984).

[30] Y. Nutku and M.P. Pavlov, Multi-Lagrangians for integrable systems, J. Math. Phys. 43 (2002) 14411459.

[31] P. Olver, Applications of Lie Groups to Differential Equations, $2^{\text {nd }}$ ed. (Springer-Verlag, New York, 1993).

[32] A.K. Prykarpatsky, O.D. Artemovych, Z. Popowicz and M.V. Pavlov, Differential-algebraic integrability analysis of the generalized Riemann type and Korteweg-de Vries hydrodynamical equations, $J$. Phys. A: Math. Theor. 43 (2010) 295205. (13pp)

[33] Y.A. Prykarpatsky, O.D. Artemovych, M. Pavlov and A.K. Prykarpatsky, The differential-algebraic and bi-Hamiltonian integrability analysis of the Riemann type hierarchy revisited, J. Math. Phys. 53 (2012) 103521; arXiv:submit/0322023 [nlin.SI] 20 Sept. 2011.

[34] Y.A. Prykarpatsky, O.D. Artemovych, M. Pavlov and A.K. Prykarpatsky, The differential-algebraic integrability analysis of symplectic and Lax type structures related with the hydrodynamic Riemann 
type systems, Rep. Math. Phys. 71 (2013) 305-351.

[35] A.K. Prykarpatsky and N.N. Bogolyubov (Jr.), Complete integrability of nonlinear systems of the hydrodynamical type, Teor. Mat. Fiz. 67 (1987) 410-425.

[36] A. Prykarpatsky and I. Mykytyuk, Algebraic Integrability of Nonlinear Dynamical Systems on Manifolds: Classical and Quantum Aspects (Kluwer, the Netherlands, 1998).

[37] A. Prykarpatski, K. Soltanov and E. Özçağ, Differential-algebraic approach to constructing representations of commuting differentiations in functional spaces and its application to nonlinear integrable dynamical systems, Commun Nonlin. Sci. Numer. Simulat. 19 (2014) 1644-1649. arXiv:submit/0729174 [nlin.SI] 1 Jun 2013.

[38] Y.A. Prykarpatsky, Finite dimensional local and nonlocal reductions of one type of hydrodynamic systems, Rep. Math. Phys. 50 (2002) 349-360.

[39] M.M. Prytula, A.K. Prykarpatsky and M.I. Vovk, On the complete integrability and linearization of a Burgers-Korteweg-de-Vries type nonlinar equation, J. Math. Sci. 167 (2010) 112-117.

[40] B. Riemann, Über die Fortpflanzung ebener Luftwellen von endlicher Schwingungsweite, in Gesammelte Mathematische Werke, Zweite Auflage, 1860, 157-175 (Dover, New York, 1953).

[41] J.F. Ritt, Differential Algebra (AMS-Colloqium Publications, vol. XXXIII, Dover, New York, 1966).

[42] M. Shubin, Lectures on Mathematical Physics (Moscow State University, Moscow, MCNMO, 2003). (in Russian)

[43] T. Tao, Nonlinear Dispersive Equations: Local and Global Analysis (American Math. Soc., Providence, 2005).

[44] E. Taflin, Analytic linearization, Hamiltonian formalism and infinite sequences of constants of motion for the Burger's equation, Phys. Rev. Lett. 47 (1981) p. 1425.

[45] H. Tasso, Hamiltonian formulation of odd Burgers hierarchy, J.Phys. Math.Gen. 29 (1996) 7779-7784.

[46] G.B. Whitham, Linear and Nonlinear Waves (John Wiley \& Sons, New York, 1974). 